\title{
Reactive Power Support from End Customer Equipment
}

\author{
Gajula Jyothi $^{1}$ - Charan Teja $S^{1}$ (D) Pradeep Kumar Yemula ${ }^{1}$
}

Received: 1 May 2017 / Accepted: 17 August 2017 / Published online: 25 September 2017

(c) Springer Nature Singapore Pte Ltd. 2017

\begin{abstract}
As a part of prosumer concept, reactive power supply using the end user equipment is evolving. Reactive power injection using end user appliances is used for voltage profile correction, reduction in losses and improvement in power factor. In a radial distribution network, when there are reactive power supplying devices available at different locations, selection of reactive power source will have greater impact on the network. So, the main focus of this paper is to find the optimal location of the bus for reactive power injection based on availability. Since the objective is to reduce losses in the network optimal location is found using the sensitivity matrix for power loss with changes in reactive power injection. Also, the system energy is energy is computed. In order to demonstrate the proposed algorithm a case study is done for IEEE 33 bus radial distribution system in MATLAB programming. Results obtained show that the reduction in losses is about $20 \%$. This proves that end user reactive power equipment is helpful in improving the network profile.
\end{abstract}

Keywords Prosumer - Reactive power support - End user equipment $\cdot$ Voltage profile

Charan Teja S

ee14resch01005@iith.ac.in

Gajula Jyothi

ee14mtech11028@iith.ac.in

Pradeep Kumar Yemula

ypradeep@iith.ac.in

1 Department of Electrical Engineering, Indian Institute of Technology Hyderabad, IITH Main Road, Near NH-65, Khandi, Telangana 502285, India

\section{Introduction}

AC systems supply or consume two kinds of power: real power and reactive power. Real power accomplishes useful work while reactive power supports the voltage profile. Voltage profile maintenance is required to ensure system reliability. In an electrical power system, it is important to control voltage for proper operation of electrical power equipment. Improper voltage levels will result in overheating of generators and motors, which consequently lead to equipment damage. Also, variation of voltage will lead to increased transmission and distribution losses. Reactive power injection is proportional to voltage. Too much of reactive power injection causes abrupt rise in voltage levels which will lead to over voltage case which is not desirable case in the power system operation. Fall in voltage levels causes when the system tries to serve more load irrespective of voltage level. So, whenever there is reduction in reactive power supply voltage will fall and in order to serve the desired load more current need the available for serving. Increase in current beyond the limits causes transmission or distribution lines to trip off due to overloading condition. Customer equipment and power systems are designed to operate within the voltage ranges, usually $\pm 5 \%$ of the nominal voltage.

At low voltages, many types of equipment perform poorly; light bulbs provide less illumination, induction motors will overheat and resulting a damaged condition, and some electronic equipment will fail to operate. High voltages leads to damage of equipment and shorten their lifetimes. On an alternating-current (AC) power system. In normal power system operation voltage is controlled by injection of reactive power. But the traditional approach is to inject the reactive power at substation level. The reactive sources at substation level are capacitor banks, FACTS 
devices etc. Since the bidirectional power flow came into reality because of smart grids. Reactive power injection at customer level instead of substation level is possible. Also, this is a cost effective method. Consumers equipment like inverters, electric vehicles etc are capable of supplying reactive power. Using these equipment reactive power can be injected into the network for improving the profile and reliability of power system.

\section{Literature Survey}

Reactive power in a power system is an important parameter since its unbalance between supply and demand may cause voltage instability, sometimes black out of the system. There are many control actions available to address the problem of reactive power supply. But most of them are costly and or not practical. Most of the control methods are available at transmission level and substation level. At the transmission level actions such as switching of transmission lines [5], using flexible Ac transmission system (FACTS) devices like static synchronous compensator (STATCOM), A static VAR compensator (SVC) and unified power flow controller (or UPFC) which are electrical devices for providing fast-acting reactive power compensation on high-voltage electricity transmission networks $[1,8,9,14]$. At sub station level synchronous condensers and shunt capacitors are used.

As technology is developing more resources are capable for provision of control actions. These make a more comprehensive reactive power control that goes all the way from the transmission system level to the end-user in smart grids, [10] Sefa et al. [13] and Dominguez-Garcia et al. [4] proposed some control techniques for providing reactive power support. Rogers et al.[11] suggested the idea of using enduser reactive-power capable devices to address low voltage problems at the transmission system level. These mitigating controls of using end-user reactive-power-capable devices are made available via smartgrid technologies. These methods can be used in the system to maintain a healthy voltage profile. Reactive power resources include inverters on solar panels, electric vehicles, uninterrupted power supply systems, inverter-based home appliances, lighting, and many other distributed sources [3, 7]. The power buffer concept proposed in $[6,7,15]$ allows the power electronics to take advantage of isolation, and presents a desired behavior to the grid. In the context of the above papers, the desired behavior is to provide reactive power injection as needed. Residential-level devices can be called upon to correct voltage violations in their local area by using secure authenticated messaging to coordinate the control. Rogers et al. $[11,12]$ just considered the reactive power resources of end-users at transmission system level.
In these papers, the control action is centralized and candidate bus placement is done only by use of sensitivity analysis. But, they do not consider the fact that end user's reactive power sources change whenever they are on or off. Aquino-Lugo et al. [2] explained little about the distribution systems, they do not explain about the process of acquiring the sensitivity matrix and reactive support grouping in the distribution systems. Also, distributed energy resources of end-users have important role in modern systems, it is better to consider other resources such as distributed generation (DG) and storage with reactive power resources. It is worth noting that there are conventional reactive power control devices such as voltage regulators that cannot be eliminated from the future systems, so they should be also considered. $\mathrm{Yu}$ et al. [16] used distributed voltage control to mitigate voltage profile, but it does not consider end-user devices as well as constant voltage DGs in their work. As mentioned in above papers, the so called central control manages systems in an integrated form and mitigates voltage problem better than other control actions. But, the communication burden of this is very high that makes it unaccepted in future smart grids. Moreover, the control systems will be out of use if control center is broken. On the other hand, in the distributed voltage control, the system is decomposed into some sub-systems that are independent and have no communication links between them. Communication burden in this control is low and is suitable for future smart grids. But, generally voltage mitigation's quality in this control action is lower than the centralized control system.

In this method, Sensitivity matrix is calculated based on real time and variable data. It can be calculated for both Power variation and voltage variation in the system for change in injected reactive power amount. Based on this sensitivity analysis we can find the behavior of the system for external reactive power injection [1]. This method can be used for distribution system, for selection of bus location using end users as reactive support. In this method time to availability of reactive power from consumers can be checked and accessible reactive power can be used to select the bus location to inject that reactive power.

\section{Problem Definition}

Reactive power has a limitation of not able to travel over long distances. Traditional reactive resources are installe at transmission and substation level, but these resources cannot contribute to reactive support at distribution side. So, the following questions arises

- How to maintain voltage levels at distribution level?

- Can consumer contribute to reactive support for correction of voltages in the system? 


\section{Solution Approach}

As part of answering the above questions a methodology or approach proposed is to use prosumer technology. Prosumer means consumer who can consume as well as produce the energy. Consumer involvement help in voltage profile correction by injecting reactive power from consumer end. In the present scenario people are converging for installation of smart inverters, batteries at their home. These devices at the residential level has the ability to provide reactive power support. Since the devices has the capability of supplying reactive power, they can be called upon to correct voltage violations in their local area and supply reactive power to grid under emergency conditions, using secure and authenticated messaging to coordinate the control. Examples for those reactive sources at domestic residential level are:

- Inverters on solar panels

- Electric vehicles(EV)

- Uninterrupted power supply systems

- Inverter-based home appliances

- Many other distributed sources

Based on the above solution approach a method is developed which is described in the following section.

\section{Description of the Method}

The backbone for this method is load flow study. Basically by modifying the load flow method the analysis is done. The following are the steps involved in injecting the reactive power using end user equipment

\section{Load Flow}

The starting point of any analysis of power system will be the computation of complex voltages at all the buses. Once the voltage magnitude and phase are computed. Once the voltages at all buses are available, power flow in lines and power at each bus are found. Load flow analysis is a computational tool for this purpose for larger systems. Load flow is normally used in planning studies when a power network is being laid or when a power network is undergoing expansion. Load flow analysis provides the following data for analysis:

1. Voltage profile of the system.

2. Power flow and losses in each transmission line.

3. Reactive power at each bus.

\section{Load Flow with Reactive Support at each Bus}

Injection of reactive power externally is going to effect the system. The effect might be rise or fall in voltage levels and losses in the system. These changes are found by running load flow. Based on the obtained power losses and voltage levels in the system, reactive power support $(d q)$ at each bus during each time instant is done for studying the effect of reactive power on system losses and voltage profile.

\section{Comparison with base Load Flow}

Effect of changing the reactive power on the system performance is tested by comparing load flow with reactive power support and base case load flow. Based on the comparison results following are computed.

- Sensitivity Matrix

- Power loss reduction Matrix

- Energy Saved

\section{Power Loss Reduction Matrix}

It is the ratio of change in power loss in the system to amount of reactive power injected in the system. Change in power loss is calculated by comparing power loss of the base case system. Using this matrix, the bus with Maximum Reduction in power loss is found and thus determining the optimal location of reactive power injection [1]. Power loss reduction matrix is also called sensitivity matrix corresponding to changes in power loss.

$S_{p} \operatorname{loss}(i, t)=\partial p_{l} \operatorname{oss}(t) / \partial q$

where:

$i$ indicates bus number.

$t$ indicates time in hours.

$\partial q$ is amount of reactive power injected at $i$ th bus.

$\partial p_{\text {loss }(t)}$ is reduction in power loss compared to base case power loss at time t,with injection of $q$ at $i$ th bus.

$S_{\text {ploss }(i, t)}$ is sensitivity vector of power loss at time $\mathrm{t}$ w.r.to $Q i$ when $Q$ is injected at $i$ th bus.

\section{Load Variation Calculator}

Load curve is a curve showing the variation of load on the system with respect to (w.r.t) time. From out of the load connected, a consumer uses different fractions of the total load at various times of the day as per his/her requirements. Since a power system has to supply load to all such consumers, the load to be supplied varies continuously with time and does not remain constant. If the load is measured (in units of power) at regular intervals of time, say, once in an hour (or half-an-hour) and recorded, a curve drawn known as the load curve. A time period of only 24 hours is considered, and the resulting load curve, which is called a Daily load curve. Using daily load curve of a particular 
day, each hour Maximum and minimum percentage variation with respect to base case is found. Using the load curve and load variation percentages a load variation calculator is used for creating some randomness in the data. So, this data is used as input load data to power flow for analysis.

$L_{t}=L_{t b} *\left[1+\frac{\operatorname{Random}\left(\beta_{(\max , t)}, \beta_{(\min , t)}\right)}{100}\right]$

where:

$L_{t}$ is Load at time t, load may be the real power $(\mathrm{P})$ or Reactive power $(\mathrm{Q})$.

$L_{t b}$ is Base load.

$\beta_{(\min , t)}$ is Minimum percentage variation w.r.to base load at time $\mathrm{t}$.

$\beta_{(\max , t)}$ is Maximum percentage variation w.r.to base load at time t.

$\operatorname{Random}\left(\beta_{(\max , t)}, \beta_{(\min , t)}\right)$ is a random function that generates value between two values $\beta_{(\max , t)}, \beta_{(\min , t)}$.

\section{Optimal Bus Location}

In radial distribution system, there will be many number of loads. In these loads some may support reactive power like smart devices and some not. Those smart devices may be available at different locations which come under different buses. This availability may differ from bus to bus and time to time. For each hour, injection of reactive power is done based on the collected information from sources at one bus. Based on load variation, amount of reactive power supplied by each bus at that hour and sensitivities of buses to injected reactive power are calculated. Obtained results will provide the bus selection for reactive power injection. This optimal bus location is decided based on objective function.

\section{Objective Function}

For a given system, effect of reactive power injection from loads on the system parameters like voltage variation at the load, voltage drop in line, losses reduction in the system needs to be studied. If the objective is to improve the voltage profile of the system, sensitivity matrix need to be built based on voltage. If the objective is to reduce the real power loss then the sensitivity matrix corresponding to power loss needs to considered. Once the sensitivity matrix is build choose the bus which gives the maximum reduction in power loss.

Figure 1 shows the flow chart of implementation of the above method. Here the input data contains the line data and bus data of the system. From the base case load flow voltage pu and the reactive powers are calculated. Whenever there is a fall in voltage or increase in real power loss then reactive power injection is used at that particular bus.

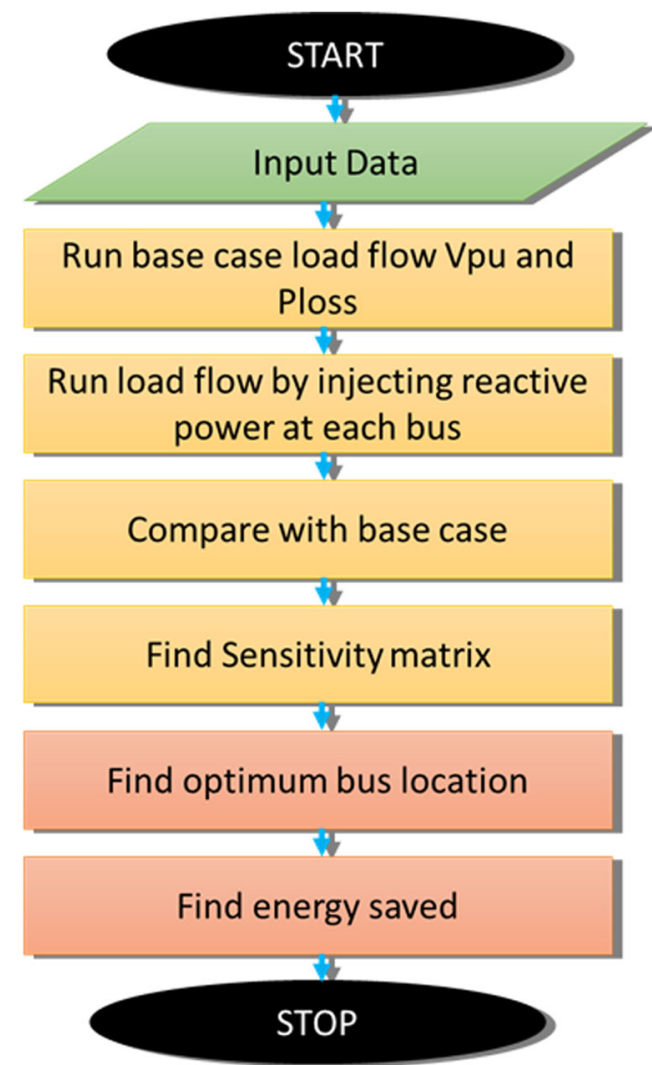

Fig. 1 Flow Chart of Implementation

In order to inject the reactive power it is assumed that every bus contains the reactive power source and consumers are willing to inject the reactive power. Now based on the voltage obtained the optimal location for injecting the reactive power is found out by forming the sensitivity matrix. Here the optimal location is found out in such a way that loss should be minimized. From the obtained bus location reactive power is injected and load flow is performed and the same is compared with the based case.

\section{Implementation Algorithm}

The following are the steps involved in implementation of the above formed method.

Step 1: Network standard details like $\mathrm{R}, \mathrm{X}$ and base case $\mathrm{P}, \mathrm{Q}$ are given as mat file. And variable input data like \% variation of load for load variation calculator and amount of reactive power externally injected (dq )are given as input data.

Step 2: Run load variation calculator with base case details as input and store those values to a matlab file which is input next program.

Step 3: Using data from step2 for time $\mathrm{t}=1$ hour, run the load flow which is considered as base case load flow and 


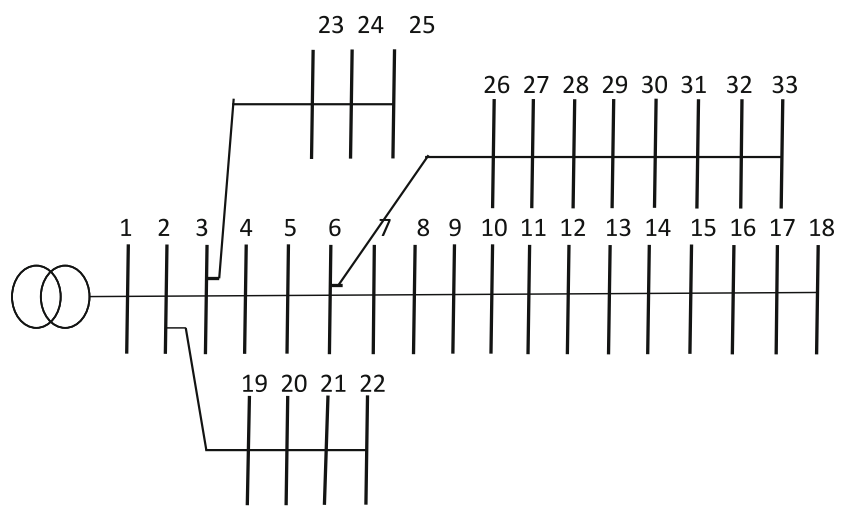

Fig. 233 bus radial system single line diagram

get the voltage profile (Vibase, $\mathrm{i}=1 \ldots . . \mathrm{n}$, where $\mathrm{n}=$ number of buses) and power loss(Ploss base) in the system.

Step 4: Assume Reactive sources are available at each bus and Assume Externally injecting Reactive power amount available is $d q$ which is given as input. With $d q$ injected at bus number $\mathrm{j}=1$, run load flow and it gives voltage profile of the system for corresponding case i.e., $V(j, i)$ and power loss in the system is $P_{l}$ oss $(j)$.

Step 5: Compare results in step 4 with base results in step3 and taking difference between these two we can form row of sensitivity matrix that is $S_{p} \operatorname{loss}(j, i)$ where $\mathrm{j}=$ bus number where $d q$ is injected and $i=1 \ldots n$. $S_{p} \operatorname{loss}(i, t)=\partial p_{l} \operatorname{oss}(t) / \partial q$ and Plossreduction $=$ Power loss in step 2-Power loss in step 3.

Step 6: Now $d q$ is injected at bus number 2 (now $\mathrm{j}=2$ ) and repeat steps step 3, step 4. Repeat for all buses.

Step 7: After completing formation of sensitivity for all cases. On which bus Maximum change in voltage and Maximum reduction in power loss is occurring, consider that bus as optimal bus location for injection $d q$ amount of reactive power for the hour $t$.
Step 8: Repeat the above steps for all time instants.

Step 9: After doing above steps calculate energy saved.

The above steps are implemented by writing a MATLAB programming and is presented as case study for IEEE 33 bus radial system.

\section{Case Study}

Figure 2 Standard IEEE 33 bus radial distribution network standard line data and bus data are used for performing the simulations.

Assumption made here is Reactive sources are available at each and every bus with same capacity assuming that capacity as $d q=0.01$ p.u i.e., $1 \mathrm{MVAr} . d q$ amount will be given as input to program. Program will run 24 cases assuming each hour of the day as each case. Initial step is to run the base case load flow. Base case load flow is done by using forward and backward sweep method. Figure 3 is the results of the base case load flow. Here voltages at each bus are considered which are used for deciding on injection of reactive power.

\section{Power loss and Optimal Bus Location}

On execution of basic load flow program following results are obtained as shown in figure at time $t=5$. Figure 4 shows the base case power loss in $\mathrm{kW}$.

From the result it is observed that Maximum reduction in power loss is $35.710 \mathrm{KW}$. This power loss is obtained by injecting reactive power of $1 \mathrm{MVAR}$ at bus 7 . Form the graph it is observed that as distance from the source increases loss in the system is also increasing. For example at 18,22 there is more power loss compared other buses since they are end buses of branches.

Fig. 3 Votlage at each bus

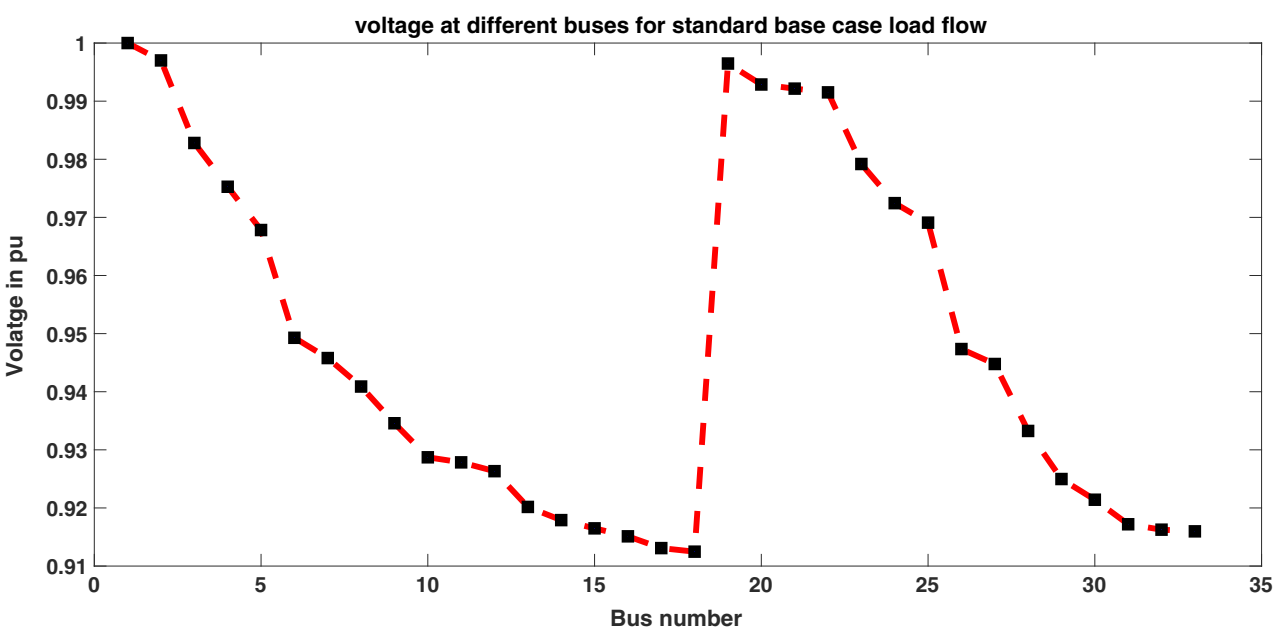


Fig. 4 Power loss in KW at each bus

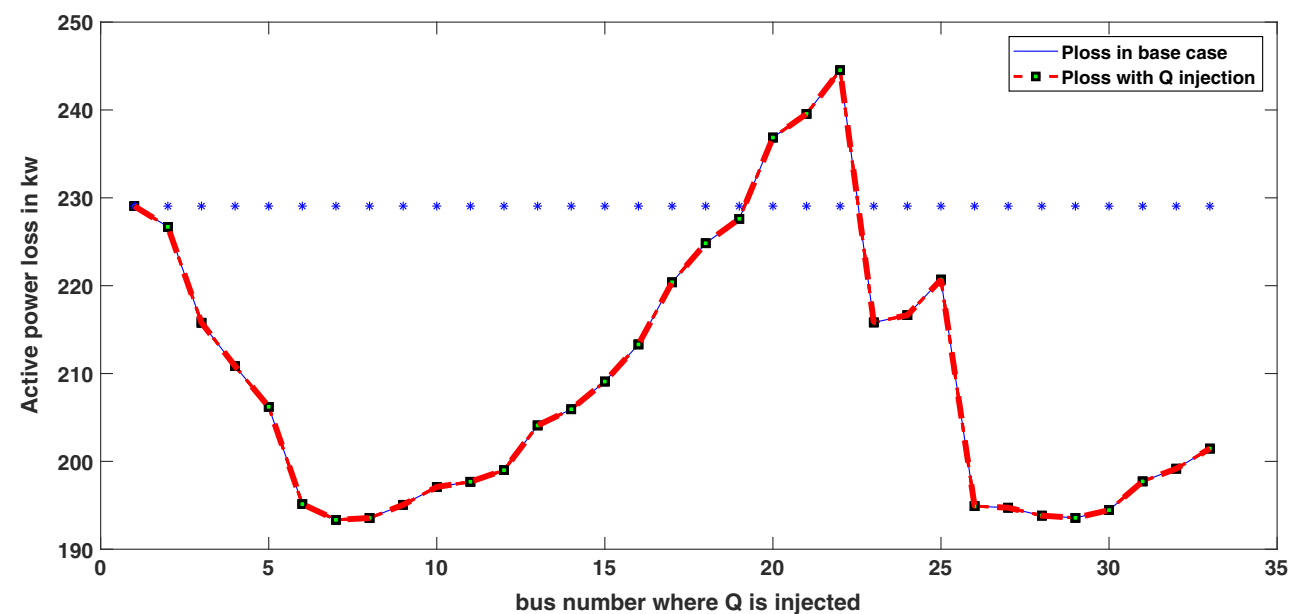

Figure 5 is the total system active power and reactive power at each hour. Also the system energy is computed for every bus. The same is depicted in the second part of Fig. 5 .

\section{Each Hour Optimum Bus Location}

Assuming same amount $d q=1$ MVAr of reactive support is available at each bus, for each hour $d q$ was injected one bus at a time and 33 cases were obtained. For each hour for each case reduction in power loss in the system when compared with base case power loss of the system is calculated. Base case for each is system without any external injection of reactive power. Based on reduction in power loss for each hour and comparing 33 cases for that hour we get best location of bus to inject $d q$ in that hour. Those best bus location have been listed for Case1. The above Table 1 provides the $24 \mathrm{hrs}$ optimal bus location data for injection of power flow. Also the reduction in power loss is also tabulated based on the injection of reactive power at the obtained optimum bus location. The values are considered by considering the constant reactive injection of 1MVAR.
Fig. 5 Total system active and reactive energy
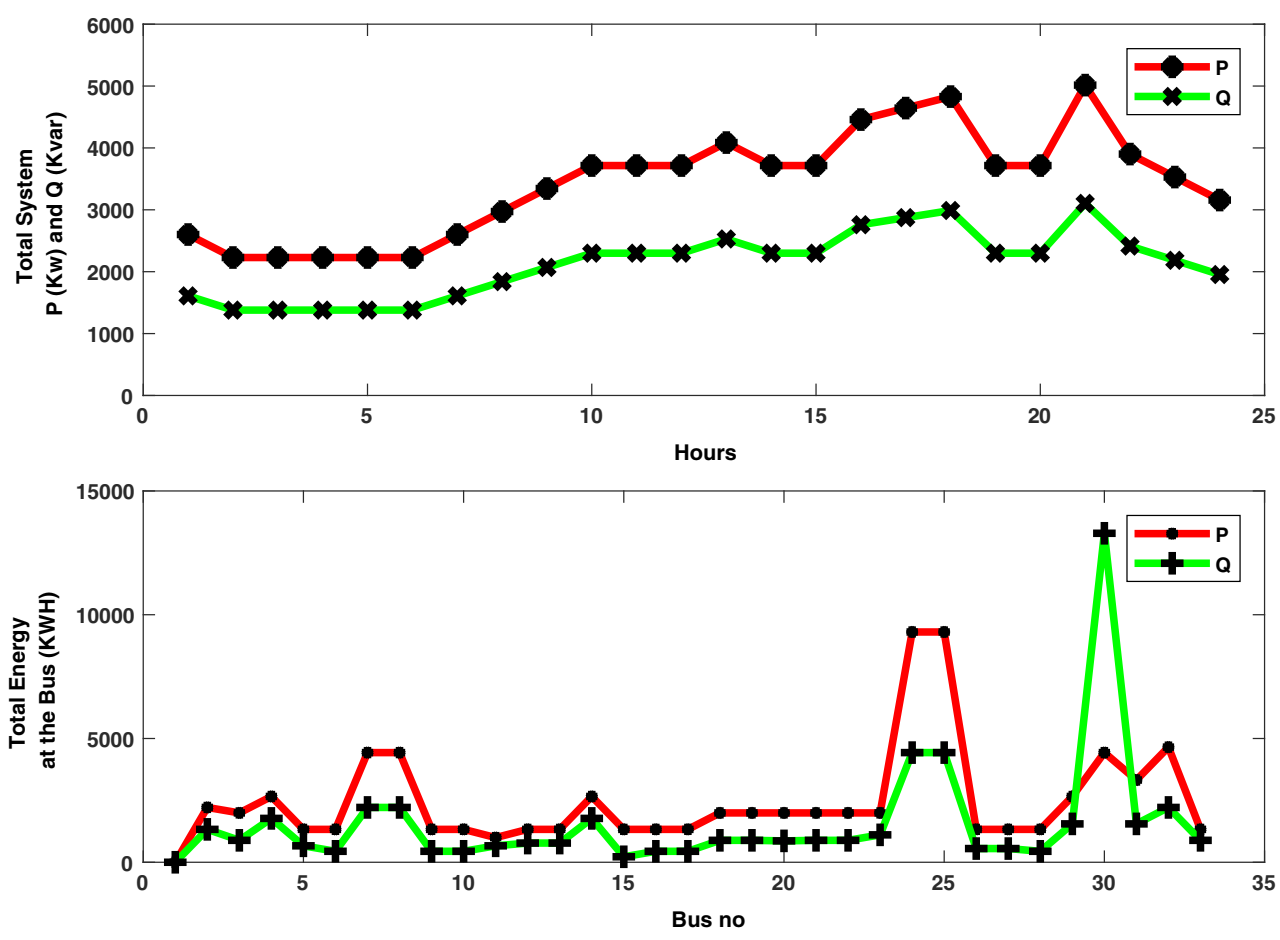
Table 1 Please write your table caption here

\begin{tabular}{|c|c|c|c|}
\hline Time in hrs & $\begin{array}{l}\text { Optimum } \\
\text { bus location }\end{array}$ & $\begin{array}{l}\text { Reactive power } \\
\text { in MVar }\end{array}$ & $\begin{array}{l}\text { Reduction in } \\
\text { Power loss in } \\
\text { Kw }\end{array}$ \\
\hline 1 & 18 & 1 & 26.201 \\
\hline 2 & 18 & 1 & 26.201 \\
\hline 3 & 18 & 1 & 22.466 \\
\hline 4 & 18 & 1 & 27.391 \\
\hline 5 & 7 & 1 & 21.39 \\
\hline 6 & 7 & 1 & 23.103 \\
\hline 7 & 7 & 1 & 30.445 \\
\hline 8 & 7 & 1 & 32.111 \\
\hline 9 & 7 & 1 & 30.361 \\
\hline 10 & 29 & 1 & 40.411 \\
\hline 11 & 29 & 1 & 40.111 \\
\hline 12 & 29 & 1 & 43.179 \\
\hline 13 & 29 & 1 & 48.868 \\
\hline 14 & 30 & 1 & 55.495 \\
\hline 15 & 29 & 1 & 47.262 \\
\hline 16 & 29 & 1 & 52.879 \\
\hline 17 & 30 & 1 & 61.525 \\
\hline 18 & 30 & 1 & 68.18 \\
\hline 19 & 29 & 1 & 53.837 \\
\hline 20 & 29 & 1 & 40.411 \\
\hline 21 & 29 & 1 & 53.633 \\
\hline 22 & 29 & 1 & 42.726 \\
\hline 23 & 7 & 1 & 23.955 \\
\hline 24 & 7 & 1 & 21.29 \\
\hline
\end{tabular}

\section{Conclusion}

This paper demonstrates how the end user equipment helps in injecting the reactive power into the distribution grid. An algorithm is proposed for selection of optimal bus location. Optimal bus selection is done considering the reduction of active power loss as an objective. From the objective sensitivity matrix is formed and the maximum reduction in losses case is considered as the optimal bus for reactive power injection. The obtained results shows about $20 \%$ reduction in losses in the system. So, it can be concluded that the concept of injecting reactive power by using end user equipment is realizable. In this paper it is considered that reactive power source is available at all buses and consumers are willing to provide reactive power support as assumptions. This work can further be extended over a large network considering only few reactive power sources are available with limited quantity. Also, this study can further extended by forming a sensitivity matrix with combination of power loss and voltage for improvement of overall network profile.

\section{References}

1. Abessi A, Vahidinasab V, Ghazizadeh MS (2016) Centralized support distributed voltage control by using end-users as reactive power support. IEEE Trans Smart Grid 7(1):178-188

2. Aquino-Lugo AA, Klump R, Overbye TJ (2011) A control framework for the smart grid for voltage support using agent-based technologies. IEEE Trans Smart Grid 2(1):173-180

3. Begović M, Pregelj A, Rohatgi A, Novosel D (2001) Impact of renewable distributed generation on power systems. In: Proceedings of the 34th Hawaii international conference on system sciences, pp 2001-2008

4. Domínguez-García AD, Hadjicostis CN, Krein PT, Cady ST (2011) Small inverter-interfaced distributed energy resources for reactive power support. In: 2011 twenty-sixth annual IEEE on applied power electronics conference and exposition (APEC). IEEE, Piscataway, pp 1616-1621

5. Hedman KW, O’Neill RP, Fisher EB, Oren SS (2009) Optimal transmission switching with contingency analysis. IEEE Trans Power Syst 24(3):1577-1586

6. Logue D, Krein PT (2000) The power buffer concept for utility load decoupling. In: 2000 IEEE 31 st annual power electronics specialists conference, 2000. PESC '00, vol 2. IEEE, Piscataway, pp 973-978

7. Logue DL, Krein PT (2001) Utility distributed reactive power control using correlation techniques. In: Sixteenth annual IEEE on applied power electronics conference and exposition, 2001. APEC 2001, vol 2. IEEE, Piscataway, pp 1294-1300

8. Ma K, Mutale J (2009) Risk and reliability worth assessment of power systems under corrective control. In: North American power symposium (NAPS), 2009. IEEE, Piscataway, pp 1-6

9. Mazi A, Wollenberg B, Hesse M (1986) Corrective control of power system flows by line and bus-bar switching. IEEE Trans Power Syst 1(3):258-264

10. Recio C (2012) The utilization of power converters in consumer products for distributed reactive power support. Thesis

11. Rogers KM, Klump R, Khurana H, Aquino-Lugo AA, Overbye TJ (2010) An authenticated control framework for distributed voltage support on the smart grid. IEEE Trans Smart Grid 1(1):40-47

12. Rogers KM, Klump R, Khurana H, Overbye TJ (2010) Smart-gridenabled load and distributed generation as a reactive resource. In: Innovative smart grid technologies (ISGT), 2010. IEEE, Piscataway, pp 1-8

13. Sefa I, Altin N, Ozdemir S (2011) An implementation of grid interactive inverter with reactive power support capability for renewable energy sources. In: 2011 international conference on power engineering, energy and electrical drives (POWERENG). IEEE, Piscataway, pp 1-6

14. Shao W, Vittal V (2006) Lp-based opf for corrective facts control to relieve overloads and voltage violations. IEEE Trans Power Syst 21(4):1832-1839

15. Weaver WW, Krein PT (2004) Mitigation of power system collapse through active dynamic buffers. In: 2004 IEEE 35th annual on power electronics specialists conference, 2004. PESC '04, vol 2. IEEE, Piscataway, pp 1080-1084

16. Yu L, Czarkowski D, De León F (2012) Optimal distributed voltage regulation for secondary networks with dgs. IEEE Trans Smart Grid 3(2):959-967 